\title{
RESEARCH
}

Open Access

\section{Systematic review of the prospective association of daily step counts with risk of mortality, cardiovascular disease, and dysglycemia}

Katherine S. Hall ${ }^{1,2^{*}}$ (D, Eric T. Hyde ${ }^{3}$, David R. Bassett ${ }^{4}$, Susan A. Carlson ${ }^{3}$, Mercedes R. Carnethon $^{5}$, Ulf Ekelund ${ }^{6}$, Kelly R. Evenson ${ }^{7}$, Deborah A. Galuska ${ }^{3}$, William E. Kraus², I-Min Lee ${ }^{8}$, Charles E. Matthews ${ }^{9}$, John D. Omura ${ }^{3}$, Amanda E. Paluch ${ }^{10}$, William I. Thomas ${ }^{11}$ and Janet E. Fulton ${ }^{3}$

\begin{abstract}
Background: Daily step counts is an intuitive metric that has demonstrated success in motivating physical activity in adults and may hold potential for future public health physical activity recommendations. This review seeks to clarify the pattern of the associations between daily steps and subsequent all-cause mortality, cardiovascular disease (CVD) morbidity and mortality, and dysglycemia, as well as the number of daily steps needed for health outcomes.

Methods: A systematic review was conducted to identify prospective studies assessing daily step count measured by pedometer or accelerometer and their associations with all-cause mortality, CVD morbidity or mortality, and dysglycemia (dysglycemia or diabetes incidence, insulin sensitivity, fasting glucose, HbA1c). The search was performed across the Medline, Embase, CINAHL, and the Cochrane Library databases from inception to August 1 , 2019. Eligibility criteria included longitudinal design with health outcomes assessed at baseline and subsequent timepoints; defining steps per day as the exposure; reporting all-cause mortality, CVD morbidity or mortality, and/or dysglycemia outcomes; adults $\geq 18$ years old; and non-patient populations.

Results: Seventeen prospective studies involving over 30,000 adults were identified. Five studies reported on allcause mortality (follow-up time 4-10 years), four on cardiovascular risk or events (6 months to 6 years), and eight on dysglycemia outcomes (3 months to 5 years). For each 1000 daily step count increase at baseline, risk reductions in all-cause mortality (6-36\%) and CVD (5-21\%) at follow-up were estimated across a subsample of included studies. There was no evidence of significant interaction by age, sex, health conditions or behaviors (e.g., alcohol use, smoking status, diet) among studies that tested for interactions. Studies examining dysglycemia outcomes report inconsistent findings, partially due to heterogeneity across studies of glycemia-related biomarker outcomes, analytic approaches, and sample characteristics.

(Continued on next page)
\end{abstract}

\footnotetext{
* Correspondence: katherine.hall@duke.edu

'Geriatric Research, Education, and Clinical Center, Durham VA Health Care System, Durham, NC, USA

${ }^{2}$ Claude D. Pepper Older Americans Independence Center, Duke Aging Center, and the Department of Medicine, Duke University, Durham, NC, USA

Full list of author information is available at the end of the article
}

(c) The Author(s). 2020 Open Access This article is licensed under a Creative Commons Attribution 4.0 International License, which permits use, sharing, adaptation, distribution and reproduction in any medium or format, as long as you give appropriate credit to the original author(s) and the source, provide a link to the Creative Commons licence, and indicate if changes were made. The images or other third party material in this article are included in the article's Creative Commons licence, unless indicated otherwise in a credit line to the material. If material is not included in the article's Creative Commons licence and your intended use is not permitted by statutory regulation or exceeds the permitted use, you will need to obtain permission directly from the copyright holder. To view a copy of this licence, visit http://creativecommons.org/licenses/by/4.0/ The Creative Commons Public Domain Dedication waiver (http://creativecommons.org/publicdomain/zero/1.0/) applies to the data made available in this article, unless otherwise stated in a credit line to the data. 


\begin{abstract}
(Continued from previous page)
Conclusions: Evidence from longitudinal data consistently demonstrated that walking an additional 1000 steps per day can help lower the risk of all-cause mortality, and CVD morbidity and mortality in adults, and that health benefits are present below 10,000 steps per day. However, the shape of the dose-response relation is not yet clear. Data are currently lacking to identify a specific minimum threshold of daily step counts needed to obtain overall health benefit.
\end{abstract}

Keywords: Physical activity, Walking, Diabetes, Prevention, Accelerometer, Physical activity guidelines, Public health

\section{Background}

The health benefits of physical activity for people of all ages, fitness levels, and sociodemographic backgrounds are well-documented [1-4]. Walking is a central component of physical activity and public health promotion efforts $[1,5]$, and daily step counts have demonstrated success as a target for achieving recommended amounts of physical activity in adults [6-8]. In addition, the expansion of wearable activity monitors and smartphones with activity-tracking capabilities onto the commercial market over the last 15 years has brought the "steps per day" activity metric into homes and healthcare systems across the world [9-11]. The increasing presence and use of self-monitoring devices and the accessibility of daily step counts as a physical activity target among the general population make it an important adjunct to current public health guidelines [7].

Despite these emerging benefits of steps for public health, recently-released guidelines for physical activity concluded there was insufficient evidence to recommend the number of daily steps needed for health. The associations of daily step counts with subsequent mortality, cardiovascular disease (CVD) risk, and type 2 diabetes were examined as part of the 2018 Physical Activity Guidelines Advisory Committee Report [6, 7]. This review was limited, however, by the small number of studies available. The authors identified 11 total articles for review, of which only 7 were longitudinal design $[6,7]$. This area of study is rapidly evolving, and a number of longitudinal studies (many with large sample sizes) have since been published. To inform future public health guidelines for physical activity, it will be important to summarize the evidence for the prospective relationship between device-measured daily step counts and health outcomes. The present review extends the previous analysis and provides an updated description of the association between daily step counts and subsequent CVD morbidity or mortality, dysglycemia, and all-cause mortality in adults and the patterns of these associations. We also investigate if these associations vary by age, sex, or moderating variables (e.g., weight status, alcohol use). The findings from this review will help form the evidence base for the number of steps per day needed for health benefit.

\section{Methods}

\section{Search strategy}

A systematic literature search was conducted in Medline, Embase, CINAHL, and the Cochrane Library from inception to August 1, 2019. The search strategy combined terms related to daily step count measured by pedometer or accelerometer with terms related to mortality, CVD, and dysglycemia (including type 2 diabetes and biomarkers such as insulin, blood glucose, HOMA, and HbA1c). A search filter limited results to randomized controlled trials and cohort studies. Articles addressing congenital heart disease were excluded.

The same strategy was used to search Medline and Embase on the OVID platform and adapted for CINAHL and Cochrane search engines. The search terms used for each database are provided in Supplemental materials. Additional citations were identified by expert consultation and review of secondary sources. The systematic review search strategy was registered with PROSPERO (CRD42020142656).

\section{Study selection}

To be included in the review, studies had to (1) use a device-based measure of daily step counts; (2) report on the association between daily step counts and mortality, CVD incidence (coronary heart disease/ischemic heart disease, coronary artery disease, stroke, heart failure, and/or metabolic syndrome), or type 2 diabetes (incidence of type 2 diabetes, dysglycemia, or changes in measures of insulin, blood glucose, HOMA, and/or HbA1c) in a prospective design; (3) be written in English; and (4) include only adults ( $\geq 18$ years of age). Studies conducted in diseased populations (e.g., heart failure, hospitalized patients, hepatitis, end-stage renal disease) were not included in this review. Study selection was performed independently by two researchers $(\mathrm{KH}$ and $\mathrm{EH}$ ), and differences relating to inclusion and exclusion criteria were resolved by consensus of the authors.

Titles and abstracts of 2144 citations were independently reviewed against inclusion criteria by $\mathrm{EH}$ and $\mathrm{KH}$. Authors excluded 1954 articles at this stage. Review of the study methods of the remaining 190 articles resulted in further exclusions. The full text of 42 articles deemed potentially relevant were retrieved for further independent 
review. Among those, 25 were excluded, leaving 17 studies included in the analysis. The accompanying chart (Fig. 1) details the results of the screening process.

\section{Data extraction}

Two authors (KH and $\mathrm{EH})$ extracted data from the 17 included studies into a preformatted table adapted for this review [12]. Descriptive information included primary outcomes; covariates included in the statistical models; sample size; characteristics of study participants - including age, sex, and other clinical characteristics; activity levels of the sample at baseline; exposure measurement including duration of monitoring period, type of device, and number of valid days required for inclusion (accelerometer studies only); statistical methods used; outcome follow-up time; and authors and year of publication. The rationale for this study was to describe and discuss the patterns of the associations between daily step counts and health outcomes, and as such, data are synthesized and presented narratively. To aid interpretation and comparison across studies, reporting of the exposure of daily step counts was standardized to 1000 steps per day difference at the baseline assessment for each health outcome. We chose the 1000 step per day increment because it required limited data manipulation to achieve harmonization across studies, as 7 out of 10 of the harmonized studies reported 1000 steps/day increments. For those studies that did not use 1000 steps per day as the base unit of analysis, we calculated standardized risk reduction scores per 1000 steps per day. For example, if the outcome measure reported was a hazard ratio with a unit of analysis was 2000 steps per day, the standardized risk reduction score was calculated as the square root of the hazard ratio and then converted to a percent risk reduction.
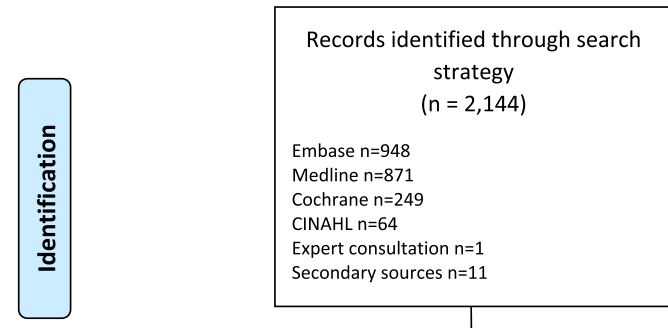

Records excluded during title/abstract screening $(n=1,954)$

Inappropriate study design $n=784$ Outcomes not relevant $n=566$

Daily steps not the exposure in analysis $n=379$ Daily steps not the
Population $n=137$ Duplicate $n=87$

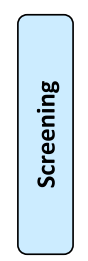

Titles or abstract potentially relevant ( $n=190)$

Language $n=1$
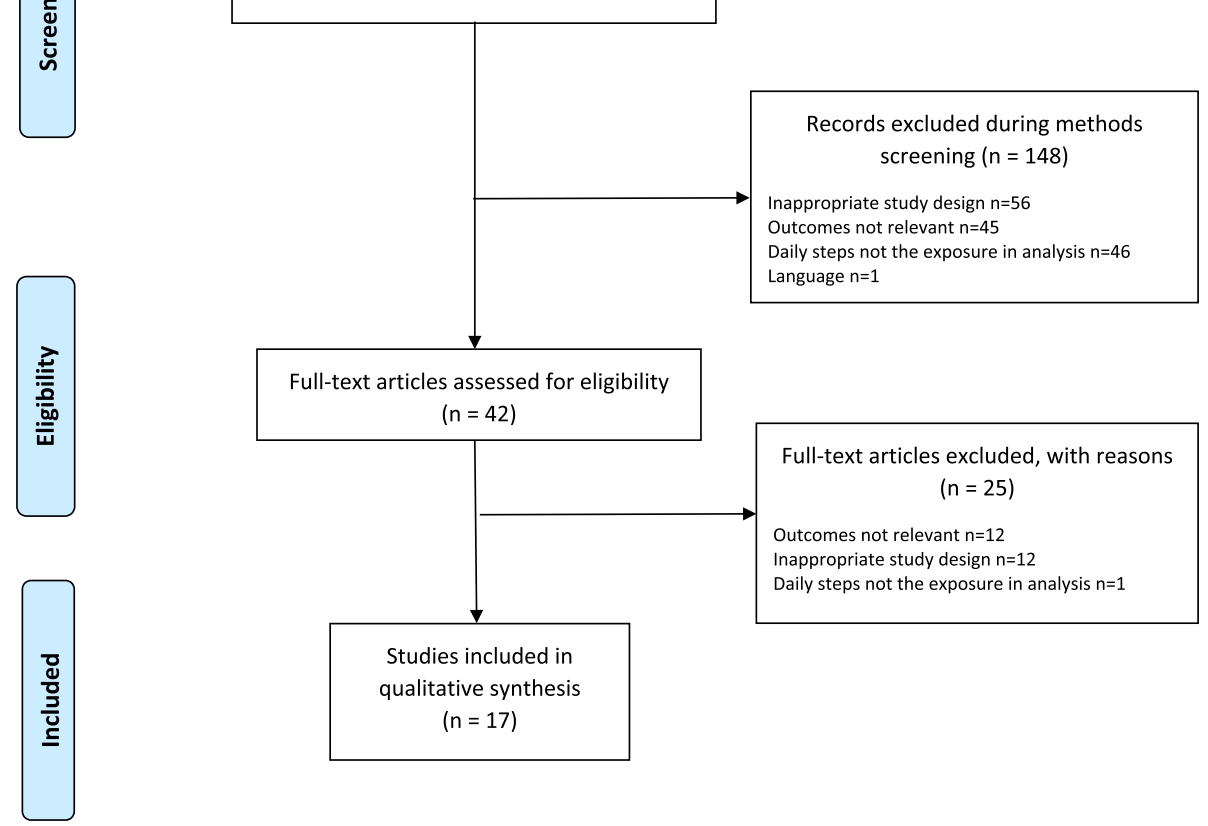

Fig. 1 Article screening process 


\section{Assessment of study quality}

$\mathrm{KH}$ and $\mathrm{EH}$ developed a quality assessment tool based on the STROBE (Strengthening the Reporting of Observational studies in Epidemiology) Statement [13] and quality assessment tool developed by Fuzeki and colleagues [14]. For each study, information on study quality was extracted by two authors (KH and $\mathrm{EH})$; differences in this assessment were discussed until consensus was reached. Study quality was determined by answers to the questions listed in Supplementary Table 1. Briefly, study quality criteria included assessment of study purpose, participant selection, measurement, reporting, statistical methods, and study limitations. Items were coded as 'yes/present' (1) or 'no/ unclear/not reported' (0). Studies scoring 8-11 were classified as high quality, those scoring 5-7 points were classified as moderate quality, and those scoring below 5 points were classified as low quality $(\max$ score $=11)$ [14].

\section{Results}

\section{Characteristics of the included studies}

Seventeen studies from 13 different cohorts were included in the systematic review; five studies assessed allcause mortality [15-19], four assessed cardiovascular events [20-23], and eight assessed dysglycemia [24-31]. Follow-up measurements of health outcomes ranged from three months [25] to 10 years [15, 19]. Sample sizes ranged from 47 [28] to 16,741 [18], with samples comprised of $46.9 \%$ female participants on average. Mean age ranged from 49.7 [27] to 78.9 [20] years; the average baseline median number of daily step counts across studies was approximately 6000 (range 2681 [20] to 10, 969 [24]). The studies were geographically diverse with participants from over 40 countries; these included Australia, the United States, the United Kingdom, South Africa, China, and Japan. Two studies of Australian adults in the AusDiab cohort reported nearly twice the number of daily steps at baseline compared to other samples (approximately 10,600 compared to approximately 5500), which may be partially due to populationlevel differences in physical activity behavior across countries. A full description of the included studies can be found in Table 1.

The methods used to measure daily step counts in the 17 studies are described in Table 2. Eleven studies measured steps with pedometers $[15,19,21,23,24,26-31]$ and six with accelerometers [16-18, 20, 22, 25]. Seven studies [16-19, 21, 22, 26] measured daily step counts only at baseline; ten studies $[15,20,23-25,27-31]$ included at least one repeated measure of step counts and subsequent health events. All studies used devices placed at the waist or hip (data not shown). Ten studies measured steps for seven consecutive days [16-23, 26, 30], two studies measured for at least 3 months [25, 31], and five studies measured for durations less than 7 days
[15, 24, 27-29]. Among studies using accelerometers, all but one [25] required at least $10 \mathrm{~h}$ per day of wear time, though the number of days required for study inclusion ranged from three [17, 20, 22] to five [16]. Two studies [23, 26], both from the NAVIGATOR trial, used multiple imputation to address missing pedometer data from the seven-day monitoring period. All other studies excluded respondents with insufficient daily step count data (range as percent of analytic sample: $<1-43 \%$ ).

The analytic methods from all included studies are described in Supplementary Table 2. The covariate measures in each study varied considerably; however, age, sex, BMI and/or waist circumference, and smoking status were the most commonly included covariate measures across all studies (Supplementary Table 2). Six studies reported sensitivity analyses excluding the first 1-3 year(s) of follow-up [15, 17-19, 22, 23]. Five studies assessed for significant differences in the association between daily step counts and outcomes by selected clinical, behavioral, and/or demographic characteristics [15, $20,23,24,26]$. Thirteen studies $[15-24,26,27,31]$ analyzed total daily step counts as a continuous variable; seven analyzed daily steps across quantiles [15-19, 22, 25]. Supplementary Table 3 shows the quality criteria scores of each study. Ten studies were of high quality $[16-19,21-24,26,30]$ and seven studies were of moderate quality $[15,20,25,27-29,31]$.

\section{Daily step counts and all-cause mortality}

Among the five studies assessing all-cause mortality, three studies of high quality $[16,18,19]$ and two studies $[15,17]$ of moderate quality reported significant associations between greater daily step counts at baseline (continuous) and less risk of all-cause mortality. All but one study [19] reported significant evidence of a linear relationship between steps per day and all-cause mortality risk (Table 3). Follow-up time across studies ranged from 4 years $[16,18]$ to 10 years $[15,19]$, and sample sizes ranged from 201 [16] to 16,741 [18] participants. In addition to analyzing daily step counts as a continuous variable, all five studies analyzed daily step counts by quantiles (Fig. 2). Each study reported significantly less risk of all-cause mortality among those in the greatest step count group compared to those in the least step count group. The average daily step counts for each quantile in Fig. 2 was plotted using quantile medians. One study compared the observed-to-expected mortality ratio across quantiles of average daily step counts [15], and four used adjusted hazard regression to compare risk of all-cause mortality in the lowest quantile of average daily step counts to all other quantiles [16-19].

Table 3 summarizes the findings of all-cause mortality studies. Based on an assumed linear association between daily step counts and mortality, for each study, reporting 


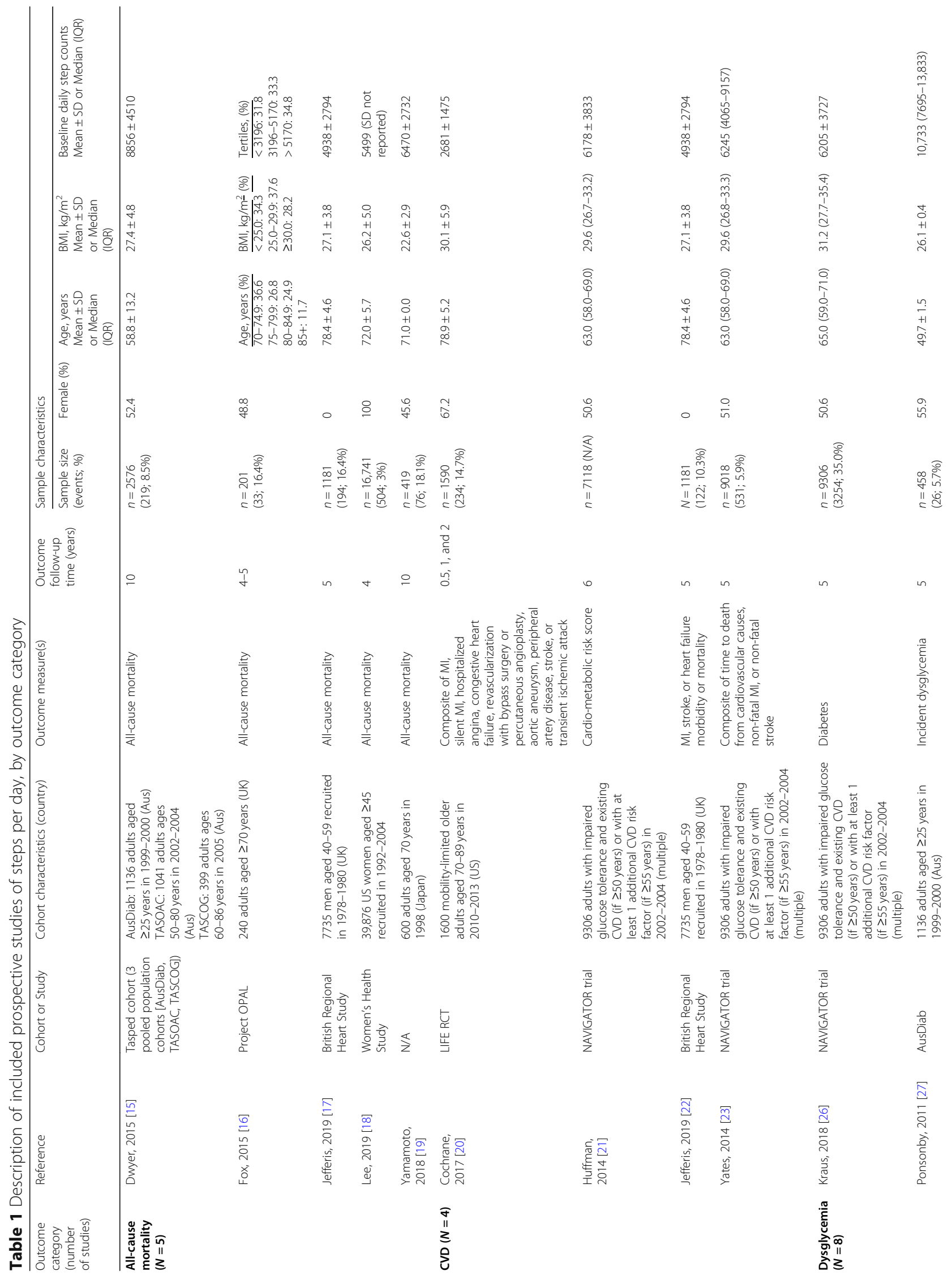




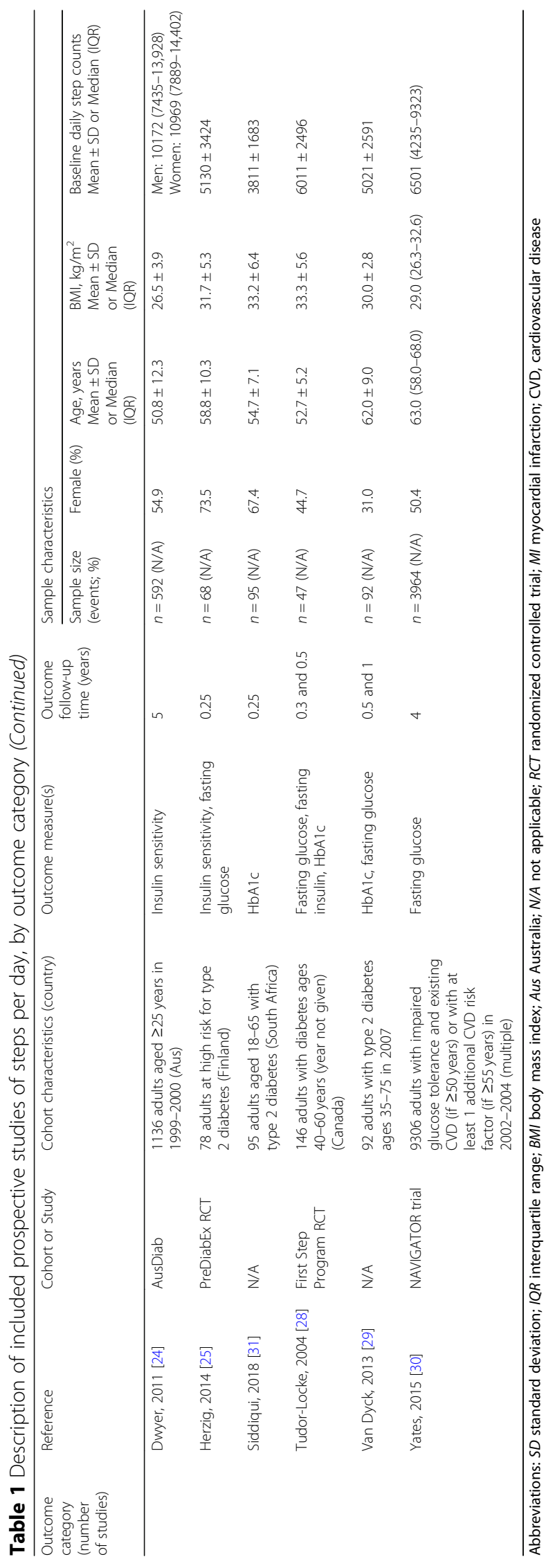




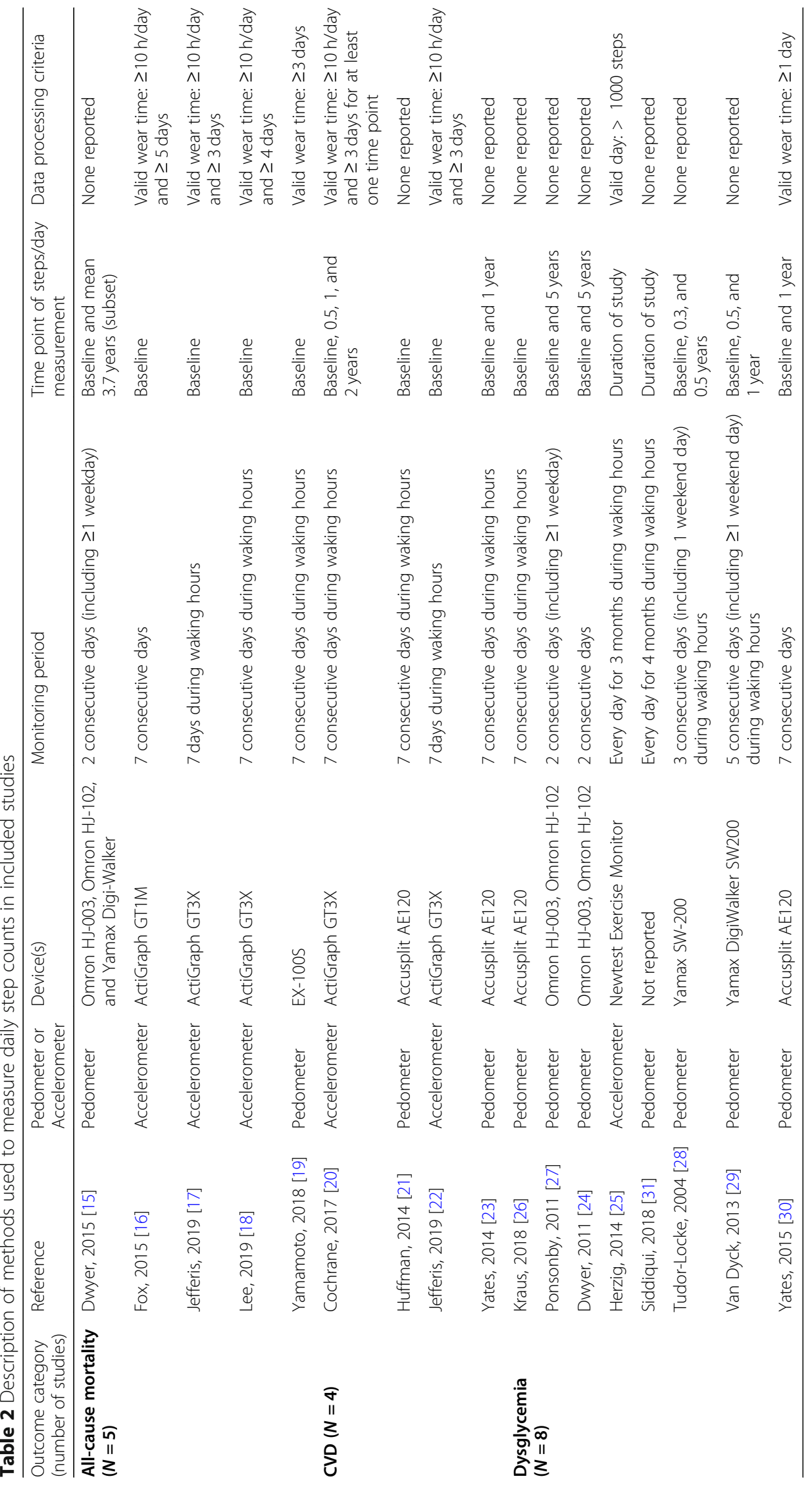




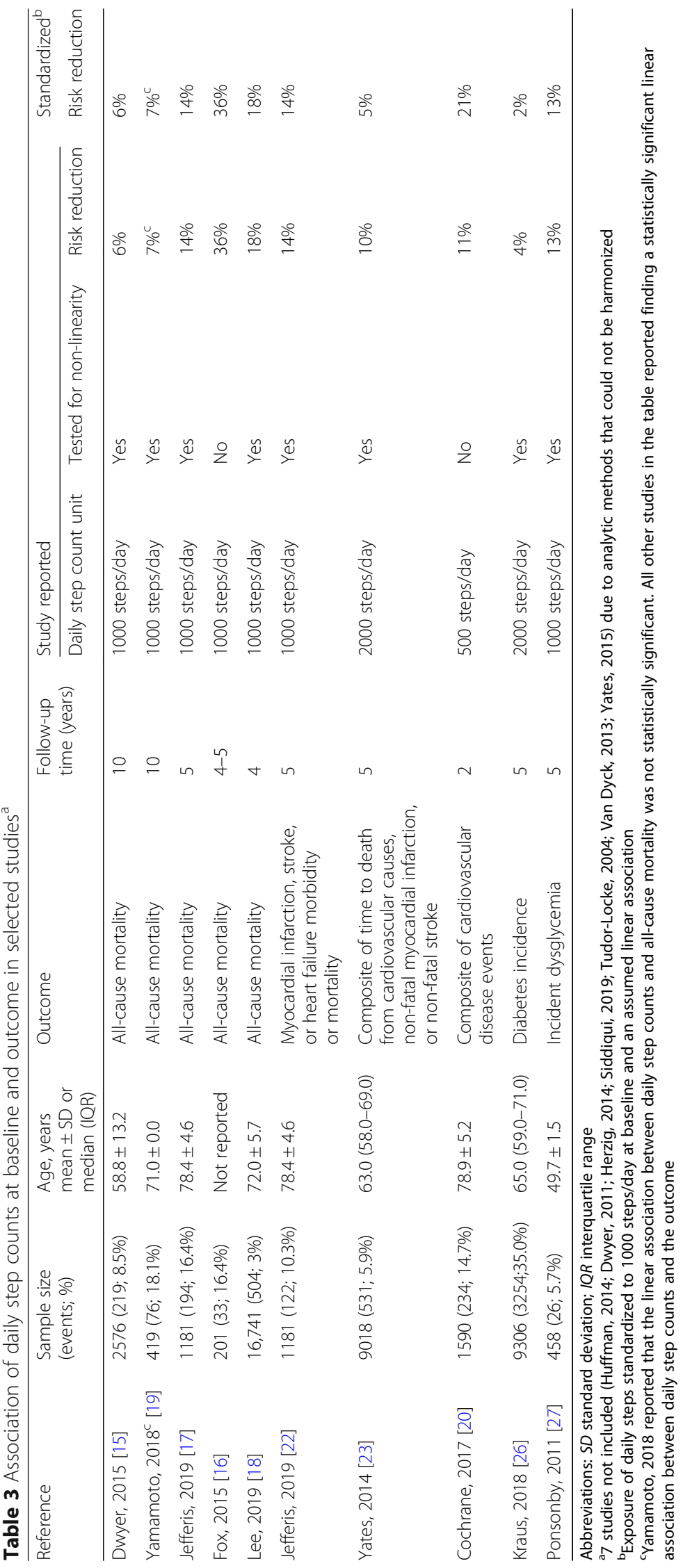




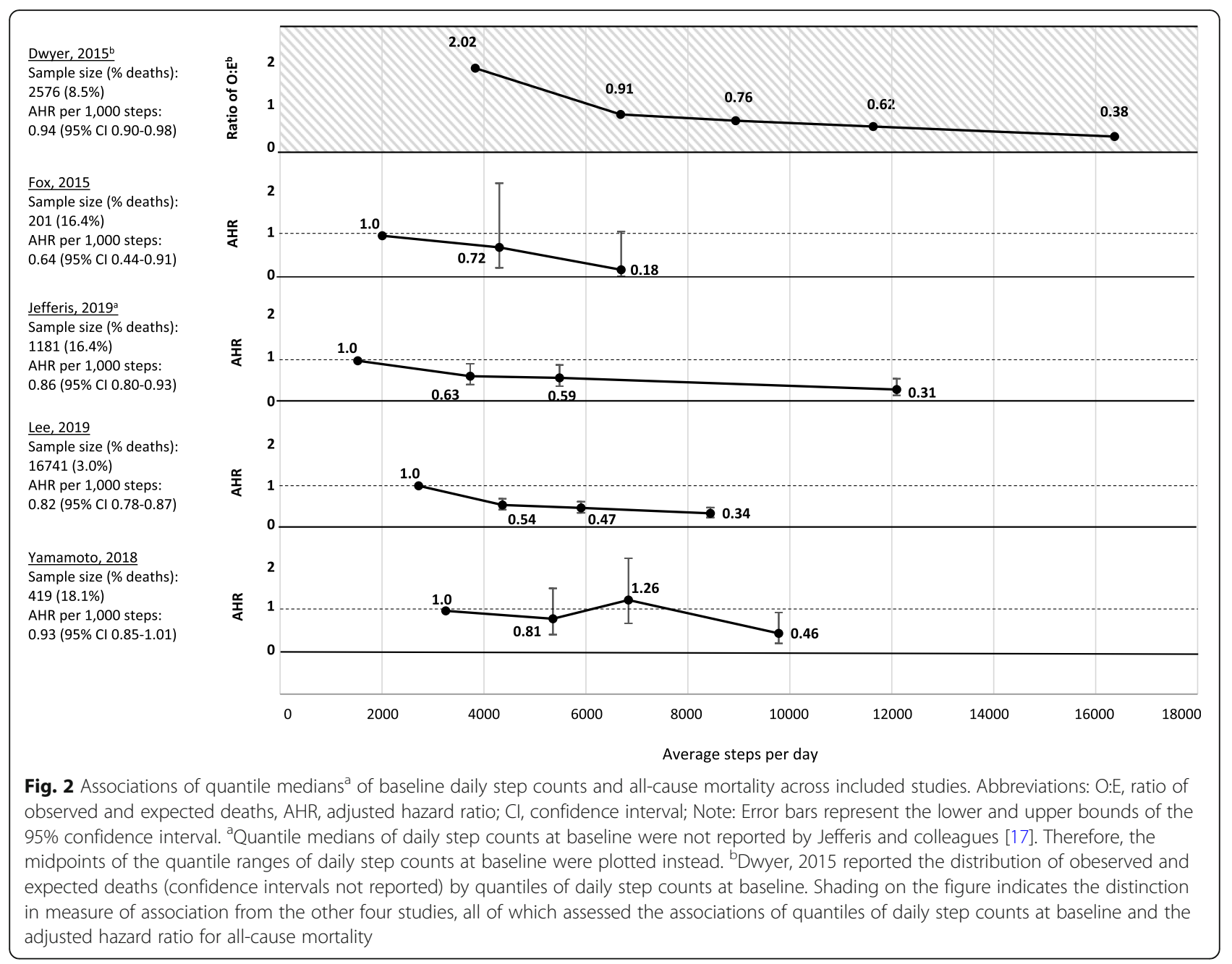

of the exposure of daily step counts was standardized to 1000 steps per day difference at the baseline assessment. The estimates for mortality are based on 21,118 participants and 103,723 person-years. The standardized risk reduction across the five studies per each 1000 daily steps increase at baseline ranged from $6 \%$ [15] to $36 \%$ [16].

One study explored prospective change in daily step counts and subsequent all-cause mortality risk among a subsample of participants [15]. The authors reported that any increase in daily step count over time, compared to no change or decrease over time, was associated with reduced mortality (AHR, 0.38, 95\% CI, 0.21-0.70, $\mathrm{P}=0.002$ ). This finding was difficult to interpret, however, because the reference group included those with either no change or reduced step counts. In addition, the authors noted more deaths were observed than expected in the reference group. Four studies [15, 17-19] conducted sensitivity analyses by removing deaths in the first 1-3 years of follow-up and reported no meaningful differences; of studies that tested for interactions, no significant interaction effects between steps and sex $[15,19]$, age $[15,17]$, or health conditions (e.g., weight status, chronic disease) and behaviors (e.g., alcohol use, diet) were reported (data not shown) $[15,17,18]$.

\section{Daily step counts and CVD morbidity or mortality}

Among the four studies assessing CVD morbidity or mortality, three studies of high quality [21-23] and one study of moderate quality [20] reported significant associations between greater daily step counts at baseline (continuous) and lower risk of CVD. These prospective studies reported CVD outcomes including calculated cardio-metabolic risk score [22], composite CVD morbidity or mortality - which included myocardial infarction, stroke, or heart failure [23], and two different composites of CVD incidence and mortality [21, 24]. The follow-up time across all four studies ranged from 6 months [21] to 6 years [22]. Sample size ranged from 1181 (a prospective cohort of men) [22] to 9018 [24] participants. 
All four studies reported significant evidence of a linear relationship between greater steps per day and lower risk of CVD morbidity or mortality. The reported risk reduction in CVD varied based on unit of difference in daily step counts. One study assessed quartiles of baseline daily step counts and reported significantly lower risk of CVD among those in the greater daily step count groups compared to those in the least step counts group [22]. Table 3 summarizes the findings of three studies $[20,22,23]$ whose analytic methods and results were comparable. The other study identified in this review was not included in this table because the health outcome (composite cardio-metabolic risk score) was not consistent with the other studies [21]. Based on an assumed linear association between daily step counts and CVD morbidity and mortality, for each study, reporting of the exposure of daily step counts was standardized to 1000 steps per day difference at the baseline assessment. The estimates for CVD are based on 11,789 participants and 54,175 person-years. The standardized risk reduction in CVD events across three studies per each 1000 daily steps increase at baseline ranged from 5\% [23] to $21 \%[20]$.

One study explored change in daily steps and subsequent CVD across repeated measurement periods [23]. Two studies conducted sensitivity analyses of previous CVD history and reported it had no effect on the association between daily step counts and incident CVD [20, 23]. Only one study conducted interaction analyses (Supplementary Table 2), and found no significant effects of age, sex, or baseline daily step counts [23].

\section{Daily step counts and dysglycemia}

Eight prospective studies [24-30] examined the relationship between daily step counts and dysglycemia; three studies were high quality $[24,26,30]$ and five were moderate quality [25, 27-29, 31]. Four studies examined associations in the context of a lifestyle intervention for adults with, or at high risk of type 2 diabetes mellitus $[25,28,29,31]$; two were conducted in the AusDiab prospective cohort [24, 27]; and two reported on the NAVIGATOR trial $[26,30]$. The four lifestyle intervention studies $[25,28,29,31]$ were small $(<100$ participants) and had short follow-up times ( $\leq 1$ year) compared to the AusDiab and NAVIGATOR studies. Three of the intervention trials targeted increased walking or daily steps $[28,29,31]$, and one targeted increased physical activity [25]. All four lifestyle intervention studies were treated as cohorts and the associations between daily step counts and the outcomes were assessed across the whole sample, regardless of study arm. These prospective studies reported on a number of dysglycemia outcomes including blood glucose levels and HbA1c, insulin resistance, 2-h glucose, insulin sensitivity (e.g., HOMAIR), and incident dysglycemia or type 2 diabetes.

Results across the longitudinal studies were mixed. Two studies examined the association between daily step counts and insulin sensitivity (assessed by HOMA-IR): one small study reported no association [25] and one large study reported a weak inverse association [24]. Six studies examined the association between daily step counts and fasting glucose or HbA1c: four reported no significant associations $[25,29-31]$, and two reported significant inverse associations [27, 28]. Two small studies with short follow-up examined daily step counts and fasting insulin; both reported no association [25, 28]. Three studies examined the association between daily step counts and 2-h glucose: one reported no association [25] and two reported weak inverse associations [28, 30]. Two larger studies with 5-year follow-up examined the association between daily step counts and incident dysglycemia or type 2 diabetes and found significantly reduced risk with greater daily step counts [26, 27]. Four studies tested interactions with confounding variables $[24,26,27,30]$ (Supplementary Table 2); they reported no significant effects of sex [24, 26, 27], age [27], or health measures $[24,27,30]$ (e.g., insulin sensitivity, smoking status, glucose tolerance, cardiovascular conditions).

Table 3 summarizes the findings of two studies [26, 27] whose analytic methods and results were comparable. The six other studies [24, 25, 28-31] identified in this review were not included in this table because no point estimate for risk reduction was reported for dysglycemia outcomes. Four studies tested linear models and all reported significant evidence of linearity for the relationship between daily step counts and dysglycemia outcomes [24, 26, 27, 30]. Based on an assumed linear association between daily step counts and dysglycemia, for each study, reporting of the exposure of daily step counts was standardized to 1000 steps per day difference at the baseline assessment. Two studies had standardized risk reduction estimates for dysglycemia outcomes: $2 \%$ [26] for incident diabetes among individuals with impaired glucose tolerance, and 13\% [27] for incident dysglycemia among individuals with normal glucose tolerance.

\section{Discussion}

This systematic review provides evidence of the benefit of increasing steps per day for health: taking more steps per day was associated with lower risk of all-cause mortality, and lower risk of CVD morbidity or mortality. Even at low levels of activity, taking an additional 1000 steps per day was associated with lower risk of all-cause mortality, and lower risk of CVD morbidity or mortality. 
These associations appear to hold across age, gender, and weight status.

This systematic review of 17 prospective studies extends the findings of the 2018 Physical Activity Guidelines Advisory Committee Report, which was limited to seven prospective studies, and provides a current summary of the prospective association between daily steps and mortality and cardiometabolic biomarkers [6, 7]. Our results are in agreement with previous studies which have reported that increases in walking (primarily based on self-report) are associated with lower risk of all-cause mortality, and CVD mortality and risk factors in adults [3, 32, 33].

We identified three new longitudinal studies of daily steps and all-cause mortality, all with large numbers, that were not included in the previous review [6]. For each 1000 step per day increase at baseline, we report a range of possible risk reductions in all-cause mortality (6-36\%) over $4-10$ years. This is a considerable expansion from the $6-7 \%$ risk reduction reported in a previous review with fewer studies [6]. Importantly, reduced risk of mortality was observed even at low levels of daily steps, below the commonly ascribed 10,000 steps per day threshold (Fig. 2). There were too few studies to test the shape of the doseresponse relationship, though our results suggest less mortality risk in adults with the greatest compared to the least daily step counts, a suggestion of "more is better" with respect to mortality risk. We identified two new longitudinal studies of daily steps and CVD morbidity or mortality that were not included in a previous review [6]. For each 1000 step per day increase at baseline, we report a range of possible risk reductions in CVD morbidity and mortality $(5-21 \%)$ over $2-5$ years. This is a considerable expansion from the $5 \%$ risk reduction reported in a previous review with fewer studies [6].

In contrast to our findings for all-cause mortality and CVD mortality and morbidity, our study could not definitively characterize the association between dysglycemia and diabetes using the eight studies identified. These inconsistent findings may be because of the heterogeneity of glycemia-related biomarker outcomes, analytic approaches, and sample characteristics. However, results from four large cohort studies with longer follow-up time do suggest a beneficial effect of increasing daily steps on incident diabetes (in adults with and without impaired glucose tolerance), fasting glucose and insulin sensitivity (in adults without impaired glucose tolerance), and 2-h glucose (in adults with impaired glucose tolerance) $[24,26,27,30]$. The findings from these four cohort studies are consistent with the findings of other systematic reviews that find that diabetes risk declines with increased levels of overall physical activity [34, 35]. The 2018 Physical Activity Guidelines Advisory Committee also concluded that there was limited evidence of an association between daily step counts and reduced risk of type 2 diabetes incidence [7, 26]; and we did not identify any new data in the present report. Additional evidence from longitudinal studies is needed to determine the association between daily steps and dysglycemia outcomes and to provide dose-response data.

Our findings appear to be robust to threats from confounding or bias. For all health outcomes, the effect of daily step counts was robust to adjustment for sociodemographic (e.g., age, sex), individual (e.g., weight status, disease history), and lifestyle characteristics (e.g., smoking, alcohol use). The generalizability of these conclusions is also supported by the representation of men and women, healthy and at-risk populations, and diverse geographical areas in this systematic review. However, only five studies (representing just two unique prospective studies) reported the racial/ethnic characteristics of the sample $[20,21,23,26,30]$ and just three studies reported the socioeconomic (e.g., education, social class, income) characteristics [16, 20,22]. Based on the available information, it appears the majority of participants in the studies included in this review were white adults, from developed countries, and of higher socioeconomic status. As a result, it is not clear how generalizable our results are to racial/ethnic minorities or low- and middle-income persons or settings.

Several methodological issues in the individual studies could have affected the findings in our review. First, we noted large disparities in the number of participants included in the analyses of daily steps counts and health outcomes compared to 1) the overall cohort sample sizes and 2) the number of adults with steps data at baseline. The issue of missing follow-up data among those participants with baseline exposure data also went largely unaddressed in these prospective studies (only 18\% reported methods for dealing with missing data; Supplementary Table 3, item \#6). Both of these issues could have biased associations or limited the generalizability of study results. Second, although the studies included in the review were all moderate-to-high quality, there was considerable heterogeneity in sample size and outcome follow-up time. Only eight studies, representing four unique prospective cohorts, reported on sample sizes $\geq 1000$ participants and follow-up time period $\geq 4$ years; the remaining studies were substantially smaller and/or had follow-up time of only 3 months to 2 years. This field would benefit from larger studies of longer duration ( $>5$ years) that provide robust estimates of the association of daily step counts with health outcomes. Finally, the cardiovascular and dysglycemia outcome measures also varied considerably across studies, with several using composite measures of differing cardiovascular events and dysglycemia markers ranging from insulin sensitivity to incident diabetes; this further complicated comparisons across studies. 
We also identified methodologic limitations specifically pertaining to exposure assessment of steps in the individual studies that warrant further discussion in the context of future research. First, we noted that different wearable devices were used to assess steps. Pedometers, while extremely useful for epidemiologic assessment, often lack the ability to store data in memory on the device, requiring participants to complete step logs [36]. Newer devices, such as accelerometers, have demonstrated excellent reliability and validity for walking, though this depends on where they are placed (usually waist-worn) and the model of the accelerometer [36, 37]. These devices often rely on proprietary algorithms to estimate step counts, making data interpretation challenging. The increasing popularity of wrist-worn consumer devices and smartphones for monitoring step counts [38] is sure to add another element of measurement variability and may introduce issues when translating research findings into public health recommendations. Second, although recommended study protocols for length and duration of accelerometer wear exist, our review found that over one-third did not carry out exposure measurement using these conventional methods [39] (Supplementary Table 3 , item \#4). These protocols include $\geq 4$ days of monitoring (pedometer and accelerometer studies), valid wear time defined as $\geq 10 \mathrm{~h} /$ day (accelerometer studies only); reporting data processing criteria (accelerometer studies only). Despite these inconsistencies, we report that as little as 2-7 days of assessment across studies was predictive of mortality and CVD outcomes. Standardized methods across studies will improve comparability, and future efforts to ensure adequate measurement and analysis of step count data will greatly improve this field of research. As activity monitoring devices are being integrated successfully into numerous large-scale prospective trials, repeated measures of step count data and subsequent health events will be available. The logistical and analytic insights from these studies could improve standardization as well as our understanding of the prospective relationship [40-42].

Our systematic review examined studies published from over the past 70 years. Despite a robust search strategy, we identified only a small number of longitudinal studies assessing daily steps and health outcomes. Many of these studies were conducted recently (past 18 years), demonstrating this as a new area of research. In addition, while 10 of the included studies included at least one repeated measure of daily step counts, only two studies prospectively examined the association of change in daily step counts and subsequent risk of the outcome [15, 23]. However, a recent study found that stability in accelerometer measured daily step counts appears stable over 2-3 years [41]. Although originally planned, we were not able to conduct meta-analyses of these studies due to the lack of standardized metrics (e.g., per 1000 steps, quantiles) and heterogeneity of study designs and populations. The lack of detailed information (e.g., point estimate and standard error) in the papers also precluded our ability to harmonize across the studies. To address these needs, we urge authors to provide detailed information in future publications on risk factor analyses and related parameters required for meta-analysis [43-45].

This is the largest systematic review to date of prospective associations between daily step counts and important health outcomes, reporting on 17 geographically diverse studies drawn from 12 unique cohorts. The 2018 Physical Activity Guidelines Advisory Committee identified a need for more evidence of the longitudinal associations between daily steps and risk of mortality, CVD, and dysglycemia. This systematic review addresses this need and identifies opportunities for additional studies to advance the field and build the evidence base around daily steps for health. Information on steps and health may be used to augment current recommendations on the amount of physical activity needed for health. Although physical activity (e.g., frequency, intensity, duration) may be easy to intuit for someone who exercises regularly, individuals who participate in activities such as gardening, house cleaning, or walking for errands may have difficulty determining the duration and intensity of these activities. Among such people, using daily steps obtained from activity trackers may be another way to promote public health guidelines and help individuals achieve the recommended amounts of physical activity. Another strength of the current review is that it investigates individual cardiometabolic biomarkers (e.g., insulin sensitivity, fasting glucose, cardio-metabolic risk score) in addition to global measures of cardiovascular and metabolic health (e.g., type 2 diabetes, CVD).

In conclusion, there was consistent evidence from longitudinal data that walking an additional 1000 steps per day can help lower the risk of all-cause mortality, and CVD morbidity and mortality in adults, and that health benefits are present below 10,000 steps per day. Our review also demonstrates a current lack of data on the relationship between step counts and subsequent health outcomes to adequately inform a daily step count guideline $[7,8]$. Additional evidence can come from completing new analyses of existing studies with the requisite exposure and outcome measures reported here, or by designing new studies that address some of the limitations noted in our review. These studies could include using standard methodologies and examine the relationship in different subgroups of the population. This additional evidence will help guide meaningful volume targets that can be used for health care, education, and behavioral interventions, and potentially inform the development of public health guidelines for steps and health. 


\section{Supplementary information}

Supplementary information accompanies this paper at https://doi.org/10. 1186/s12966-020-00978-9.

\section{Additional file 1.}

\section{Abbreviations}

CVD: Cardiovascular disease; BMI: Body mass index; AHR: Adjusted hazard ratio

\section{Acknowledgements}

Not applicable.

\section{Authors' contributions}

$\mathrm{KH}$ led the review process, drafting of the manuscript, and incorporating all feedback. EH assisted in performing the review, drafting the manuscript, and administrative duties. JF, SC, and DG provided expert consultation and intellectual feedback. BT designed the search strategy and performed the literature search. All other authors provided feedback on the review process and manuscript drafting. All authors read and approved the final manuscript.

\section{Funding}

IML is supported in part by the National Cancer Institute (CA182913). KRE is supported by the NIH National Cancer Institute and Office of the Director (1R01CA227122). KSH is supported by the National Institutes of Health, Claude D. Pepper Older Americans Independence Center (NIA P30AG028716) and the Department of Veterans Affairs, Veterans Health Administration, Office of Research and Development, Rehabilitation Research and Development Service (2RX001316). The findings and conclusions in this report are those of the authors and do not necessarily represent the official position or policy of the Centers for Disease Control and Prevention, the Department of Veterans Affairs, or the United States government.

\section{Availability of data and materials}

Data sharing is not applicable to this article as no datasets were generated or analyzed during the current study.

\section{Ethics approval and consent to participate}

Not applicable.

\section{Consent for publication}

Not applicable.

\section{Competing interests}

None to report.

\section{Author details}

${ }^{1}$ Geriatric Research, Education, and Clinical Center, Durham VA Health Care System, Durham, NC, USA. ${ }^{2}$ Claude D. Pepper Older Americans Independence Center, Duke Aging Center, and the Department of Medicine, Duke University, Durham, NC, USA. 'Division of Nutrition, Physical Activity, and Obesity, National Center for Chronic Disease Prevention and Health Promotion, Centers for Disease Control and Prevention, Atlanta, GA, USA. ${ }^{4}$ Department of Kinesiology, Recreation, and Sport Studies, The University of Tennessee, Knoxville, TN, USA. ${ }^{5}$ Department of Preventive Medicine, Northwestern University, Chicago, IL, USA. 'Department of Sport Medicine, Norwegian School of Sport Sciences, Oslo, Norway and Department of Chronic Diseases and Ageing, Norwegian Institute of Public Health, Oslo, Norway. ${ }^{7}$ Department of Epidemiology, Gillings School of Global Public Health, University of North Carolina - Chapel Hill, Chapel Hill, NC, USA. ${ }^{8}$ Brigham and Women's Hospital, Harvard Medical School; Harvard T.H. Chan School of Public Health, Boston, MA, USA. ${ }^{9}$ Metabolic Epidemiology Branch, Division of Cancer Epidemiology and Genetics, National Cancer Institute, Rockville, MD, USA. ${ }^{10}$ Department of Kinesiology, Institute for Applied Life Sciences, University of Massachusetts, Amherst, MA, USA. ${ }^{11}$ Office of Library Science, Office of Science, Centers for Disease Control and Prevention, Atlanta, GA, USA.
Received: 20 December 2019 Accepted: 28 May 2020

Published online: 20 June 2020

\section{References}

1. U.S. Department of Health and Human Services. Step It Up! The Surgeon General's Call to Action to Promote Walking and Walkable Communities. Washington, DC: U.S. Department of Health and Human Services, Office of the Surgeon General; 2015.

2. Lee IM, Buchner DM. The importance of walking to public health. Med Sci Sports Exerc. 2008;40(7):S512-8.

3. Oja P, Kelly P, Murtagh EM, Murphy MH, Foster C, Titze S. Effects of frequency, intensity, duration and volume of walking interventions on CVD risk factors: a systematic review and meta-regression analysis of randomised controlled trials among inactive healthy adults. Br J Sports Med. 2018;52(12): 769-75.

4. Ekelund U, Tarp J, Steene-Johannessen J, Hansen BH, Jefferis B, Fagerland MW, et al. Dose-response associations between accelerometry measured physical activity and sedentary time and all cause mortality: systematic review and harmonised meta-analysis. BMJ. 2019;366:14570.

5. Harris T, Limb ES, Hosking F, Carey I, DeWilde S, Furness C, et al. Effect of pedometer-based walking interventions on long-term health outcomes: prospective 4-year follow-up of two randomised controlled trials using routine primary care data. PLoS Med. 2019;16(6):e1002836.

6. Kraus WE, Janz KF, Powell KE, Campbell WW, Jakicic JM, Troiano RP, et al. Daily step counts for measuring physical activity exposure and its relation to health. Med Sci Sports Exerc. 2019;51(6):1206-12.

7. 2018 Physical Activity Guidelines Advisory Committee. 2018 physical activity guidelines advisory committee scientific report. Washington, DC: U.S. Department of Health and Human Services; 2018.

8. Piercy KL, Troiano RP, Ballard RM, Carlson SA, Fulton JE, Galuska DA, et al. The physical activity guidelines for Americans. JAMA. 2018;320(19):2020-8.

9. Bunn JA, Navalta JW, Fountaine CJ, Reece JD. Current state of commercial wearable technology in physical activity monitoring 2015-2017. Int J Exerc Sci. 2018;11(7):503-15.

10. Thompson WR. Worldwide survey of fitness trends for 2019. ACSMs Health Fit J. 2018;22(6):10-7.

11. Lamkin P. Smartwatch popularity booms with fitness trackers on the slide. Forbes. Accessed 22 Feb 2018. Available at: https://www.forbes.com/sites/ paullamkin/2018/02/22/smartwatch-popularity-booms-with-fitness-trackerson-the-slide/\#5c694b6e7d96.

12. Brocklebank LA, Falconer CL, Page AS, Perry R, Cooper AR. Accelerometermeasured sedentary time and cardiometabolic biomarkers: a systematic review. Prev Med. 2015;76:92-102.

13. von Elm E, Altman DG, Egger M, Pocock SJ, Gotzsche PC, Vandenbroucke $J P$, et al. The strengthening the reporting of observational studies in epidemiology (STROBE) statement: guidelines for reporting observational studies. PLoS Med. 2007;4(10):e296.

14. Fuzeki E, Engeroff T, Banzer W. Health benefits of light-intensity physical activity: a systematic review of accelerometer data of the National Health and nutrition examination survey (NHANES). Sports Med. 2017;47(9):1769-93.

15. Dwyer T, Pezic A, Sun C, Cochrane J, Venn A, Srikanth V, et al. Objectively measured daily steps and subsequent long term all-cause mortality: the tasped prospective cohort study. PLoS One. 2015;10(11):e0141274.

16. Fox KR, Ku PW, Hillsdon M, Davis MG, Simmonds BA, Thompson JL, et al. Objectively assessed physical activity and lower limb function and prospective associations with mortality and newly diagnosed disease in UK older adults: an OPAL four-year follow-up study. Age Ageing. 2015;44(2): 261-8.

17. Jefferis BJ, Parsons TJ, Sartini C, Ash S, Lennon LT, Papacosta O, et al. Objectively measured physical activity, sedentary behaviour and all-cause mortality in older men: does volume of activity matter more than pattern of accumulation? Br J Sports Med. 2019;53:1013-20.

18. Lee IM, Shiroma EJ, Kamada M, Bassett DR, Matthews CE, Buring JE. Association of step volume and intensity with all-cause mortality in older women. JAMA Intern Med. 2019;179(8):1105-12.

19. Yamamoto N, Miyazaki H, Shimada M, Nakagawa N, Sawada SS, Nishimuta $M$, et al. Daily step count and all-cause mortality in a sample of Japanese elderly people: a cohort study. BMC Public Health. 2018;18(1):540.

20. Cochrane SK, Chen SH, Fitzgerald JD, Dodson JA, Fielding RA, King AC, et al. Association of accelerometry-measured physical activity and cardiovascular 
events in mobility-limited older adults: the LIFE (lifestyle interventions and Independence for elders) study. J Am Heart Assoc. 2017;6(12):e007215.

21. Huffman KM, Sun JL, Thomas L, Bales CW, Califf RM, Yates T, et al. Impact of baseline physical activity and diet behavior on metabolic syndrome in a pharmaceutical trial: results from NAVIGATOR. Metabolism. 2014;63(4):554-61.

22. Jefferis BJ, Parsons TJ, Sartini C, Ash S, Lennon LT, Papacosta O, et al. Does total volume of physical activity matter more than pattern for onset of CVD? A prospective cohort study of older British men. Int J Cardiol. 2019; 278:267-72.

23. Yates T, Haffner SM, Schulte PJ, Thomas L, Huffman KM, Bales CW, et al. Association between change in daily ambulatory activity and cardiovascular events in people with impaired glucose tolerance (NAVIGATOR trial): a cohort analysis. Lancet. 2014;383(9922):1059-66.

24. Dwyer T, Ponsonby AL, Ukoumunne OC, Pezic A, Venn A, Dunstan D, et al. Association of change in daily step count over five years with insulin sensitivity and adiposity: population based cohort study. BMJ. 2011;342:C7249.

25. Herzig KH, Ahola R, Leppäluoto J, Jokelainen J, Jämsä T, KeinänenKiukaanniemi S. Light physical activity determined by a motion sensor decreases insulin resistance, improves lipid homeostasis and reduces visceral fat in high-risk subjects: PreDiabEx study RCT. Int J Obes (Lond). 2014;38:1089.

26. Kraus WE, Yates T, Tuomilehto J, Sun JL, Thomas L, McMurray JJV, et al. Relationship between baseline physical activity assessed by pedometer count and new-onset diabetes in the NAVIGATOR trial. BMJ Open Diabetes Res Care. 2018;6(1):e000523.

27. Ponsonby AL, Sun C, Ukoumunne OC, Pezic A, Venn A, Shaw JE, et al. Objectively measured physical activity and the subsequent risk of incident dysglycemia: the Australian diabetes, obesity and lifestyle study (AusDiab). Diabetes Care. 2011;34(7):1497-502.

28. Tudor-Locke C, Bell RC, Myers AM, Harris SB, Ecclestone NA, Lauzon N, et al. Controlled outcome evaluation of the first step program: a daily physical activity intervention for individuals with type II diabetes. Int J Obes. 2004; 28(1):113-9.

29. Van Dyck D, De Greef K, Deforche B, Ruige J, Bouckaert J, Tudor-Locke CE, et al. The relationship between changes in steps/day and health outcomes after a pedometer-based physical activity intervention with telephone support in type 2 diabetes patients. Health Educ Res. 2013;28(3):539-45.

30. Yates T, Davies MJ, Haffner SM, Schulte PJ, Thomas L, Huffman KM, et al. Physical activity as a determinant of fasting and 2-h post-challenge glucose: a prospective cohort analysis of the NAVIGATOR trial. Diabet Med. 2015; 32(8):1090-6.

31. Siddiqui MA, Bhana S, Daya R. The relationship between objective measured physical activity and parameters of disease control in an African population of type 2 diabetes mellitus. JEMDSA. 2018;23(3):80-5.

32. Kelly P, Kahlmeier S, Gotschi T, Orsini N, Richards J, Roberts N, et al. Systematic review and meta-analysis of reduction in all-cause mortality from walking and cycling and shape of dose response relationship. Int I Behav Nutr Phys Act. 2014;11:132

33. Stamatakis E, Kelly P, Strain T, Murtagh EM, Ding D, Murphy MH. Self-rated walking pace and all-cause, cardiovascular disease and cancer mortality: individual participant pooled analysis of 50225 walkers from 11 population British cohorts. Br J Sports Med. 2018;52(12):761-8.

34. Reiner M, Niermann C, Jekauc D, Woll A. Long-term health benefits of physical activity-a systematic review of longitudinal studies. BMC Public Health. 2013;13(1):813.

35. Jefferis BJ, Whincup PH, Lennon L, Wannamethee SG. Longitudinal associations between changes in physical activity and onset of type 2 diabetes in older British men: the influence of adiposity. Diabetes Care. 2012;35(9):1876-83.

36. Bassett DR Jr, Toth LP, LaMunion SR, Crouter SE. Step counting: a review of measurement considerations and health-related applications. Sports Med. 2017;47(7):1303-15.

37. Strath SJ, Kaminsky LA, Ainsworth BE, Ekelund U, Freedson PS, Gary RA, et al. Guide to the assessment of physical activity: clinical and research applications: a scientific statement from the American Heart Association. Circulation. 2013;128(20):2259-79.

38. Amagasa S, Kamada M, Sasai H, Fukushima N, Kikuchi H, Lee IM, et al. How well iphones measure steps in free-living conditions: cross-sectional validation study. JMIR Mhealth Uhealth. 2019;7(1):e10418.
39. Tudor-Locke C, Camhi SM, Troiano RP. A catalog of rules, variables, and definitions applied to accelerometer data in the National Health and nutrition examination survey, 2003-2006. Prev Chronic Dis. 2012;9:E113.

40. Lee IM, Shiroma EJ, Evenson KR, Kamada M, LaCroix AZ, Buring JE. Using devices to assess physical activity and sedentary behavior in a large cohort study, the Women's health study. J Meas Phys Behav. 2018;1(2):60-9.

41. Keadle SK, Shiroma EJ, Kamada M, Matthews CE, Harris TB, Lee IM. Reproducibility of accelerometer-assessed physical activity and sedentary time. Am J Prev Med. 2017;52(4):541-8.

42. Troiano RP, McClain JJ, Brychta RJ, Chen KY. Evolution of accelerometer methods for physical activity research. Br J Sports Med. 2014;48(13):1019-23.

43. Stroup DF, Berlin JA, Morton SC, Olkin I, Williamson GD, Rennie D, et al. Meta-analysis of observational studies in epidemiology: a proposal for reporting. Meta-analysis of observational studies in epidemiology (MOOSE) group. JAMA. 2000;283(15):2008-12.

44. Mueller M, D'Addario M, Egger M, Cevallos M, Dekkers O, Mugglin C, et al. Methods to systematically review and meta-analyse observational studies: a systematic scoping review of recommendations. BMC Med Res Methodol. 2018;18(1):44.

45. Uman LS. Systematic reviews and meta-analyses. J Can Acad Child Adolesc Psychiatry. 2011;20(1):57-9.

\section{Publisher's Note}

Springer Nature remains neutral with regard to jurisdictional claims in published maps and institutional affiliations.
Ready to submit your research? Choose BMC and benefit from:

- fast, convenient online submission

- thorough peer review by experienced researchers in your field

- rapid publication on acceptance

- support for research data, including large and complex data types

- gold Open Access which fosters wider collaboration and increased citations

- maximum visibility for your research: over $100 \mathrm{M}$ website views per year

At BMC, research is always in progress.

Learn more biomedcentral.com/submissions 